\title{
An Induction Heating Analysis with Consideration of Temperature Dependent B-H Curves and Change in Phase Transformation under Rapid Heating
}

\author{
Hirohisa TAKEUCHI* and Yasuhiro YOGO \\ Toyota Central R\&D Lab., Inc., 41-1, Yokomichi, Nagakute, Aichi, 480-1192 Japan. \\ (Received on August 6, 2018; accepted on November 19, 2018)
}

\begin{abstract}
Induction heating $(\mathrm{IH})$ is commonly used for heating and heat treatment. An accurate prediction of temperature distribution is required to optimize the heating parameters, such as the heating coil dimensions, coil current, and heating time, without trial and error. In this study, temperature-dependent B-H curves and changes in phase transformation under rapid heating were measured and used for $\mathrm{IH}$ analysis to improve the prediction accuracy. $\mathrm{IH}$ experiments were also carried out to confirm the accuracy of the analysis. Consideration of the temperature-dependent B-H curves improved the prediction accuracy up to the Curie temperature, and consideration of changes in phase transformation temperature under rapid heating improved the accuracy for temperatures above the $A c_{1}$ temperature. The maximum error between the experimental and calculation results for a columnar test piece was approximately $25 \mathrm{~K}$, when the experimental temperature was $1300 \mathrm{~K}$. This error is 9 times smaller than that obtained with a conventional analysis method. Good agreement was also obtained between the experimental and calculated values for a test piece with a convex projection, for which the temperature distribution and evolution are complex.
\end{abstract}

KEY WORDS: induction heating analysis; simulation; B-H curve; phase transformation; rapid heating.

\section{Introduction}

Induction heating $(\mathrm{IH})$ is a popular heating method. In 1969 , IH was widely used in industry ${ }^{1)}$ due to its high efficiency. Various kinds of industrial applications have been reported, including hardening, ${ }^{2)}$ hot forging ${ }^{3)}$, die casting ${ }^{4)}$, liquid-solid casting, ${ }^{5)}$ shrinkage fitting, ${ }^{6)}$ brazing, ${ }^{7)}$ and the Czochralski method for crystal growth. ${ }^{8)}$ Metal-metal bonding, ${ }^{9}$ silicon-grass bonding, ${ }^{10)}$ and bonding and dismantling processes $^{11)}$ have been conducted with IH. Recently, IH has been combined with other processes to improve material characteristics; for example, it was combined with gas nitriding to improve tribological properties ${ }^{12)}$ and with shot peening to increase pitting potential. ${ }^{13)}$ In addition, IH has been applied in the disassembly of mobile phone terminals $^{14)}$ and the extraction of pectin. ${ }^{15)}$

In $\mathrm{IH}$, the heating parameters (e.g., heating coil dimensions, coil current, heating time, and clearance between a workpiece and a coil) strongly influence the temperature distribution and heating rate. Heating parameters are usually optimized by trial and error, which is time-consuming and expensive. Kawasaki suggested using IH simulation for optimization. $^{16)}$

Miyachika et al. ${ }^{17)}$ simulated temperature evolution and stresses during $\mathrm{IH}$, and the maximum error between simulation and experimental results was approximately

\footnotetext{
* Corresponding author: E-mail: take-hrhs@mosk.tytlabs.co.jp DOI: https://doi.org/10.2355/isijinternational.ISIJINT-2018-552
}

$150 \mathrm{~K}$ when a workpiece was $973 \mathrm{~K}$. Takagaki and Toi ${ }^{18)}$ conducted IH simulations coupled with thermal elastoviscoplasticity damage and phase transformation, and a result showed good agreement with a result of Miyachika et al. ${ }^{17)}$ In both paper, details of magnetization characteristic which considered in simulations were not mentioned. The flamework to consider nonlinear magnetization characteristics for IH simulation was suggested by Ciric et al., ${ }^{19)}$ but an accuracy of simulation was not reported.

Accurate material properties are required to accurately simulate physical phenomena. In IH simulations, the magnetization characteristic is especially important because it is the predominant property affecting heat generation. As reported by Ikuta and Horino, ${ }^{20)}$ magnetic field dependence of magnetization characteristic is shall be considered in the simulation; this is equivalent to considering the magnetization characteristic as B-H curves. Maten and Melissen reported that the magnetization characteristic changes drastically close to the Curie point $\left(\mathrm{T}_{\mathrm{C}}\right)$, and thus the temperature dependence of the magnetic characteristic has to be taken into account. ${ }^{21)}$ Accordingly, it is essential to implement temperature-dependent $\mathrm{B}-\mathrm{H}$ curves from room temperature to $\mathrm{T}_{\mathrm{C}}$ for accurate simulation.

Moreover, phase transformation temperatures are influenced by the heating rate, as reported by Miwa and Iguchi. ${ }^{22}$ This phenomenon has to be considered in IH simulations because an endothermal reaction occurs during the phase transformation from ferrite to austenite phases due to latent heat. The heating rate thus changes the temperature range in 
which temperature evolution is halted by latent heat.

The rest of this study is organized as follows. Section 2 describes measurements of temperature-dependent B-H curves and changes in phase transformation temperature under rapid heating. The measurement data are discussed in Section 3 and applied to IH simulation in Section 4. Experimental and calculation results of IH are compared to confirm the accuracy of the simulation in Section 5. Conclusions are given in Section 6.

\section{Experimental Method}

\subsection{B-H Curve Measurement}

A schematic diagram of the measurement method and the test piece are shown in Fig. 1. The test piece was made of medium carbon steel (JIS S45C), which is equivalent to ISO C45. The chemical composition of the JIS S45C used in this study is shown in Table 1. Recently, a measurement method for temperature-dependent B-H curves was developed by Takeuchi et al. ${ }^{23)}$ In a conventional measurement method of B-H curves, the measurable temperature range is limited to below $473 \mathrm{~K}\left(200^{\circ} \mathrm{C}\right)$ due to the lack of thermal resistance in the wires used for measurement. Takahashi et al. also developed a measurement method for temperaturedependent B-H curves. ${ }^{24)}$ In their method, a polyester copper wire was applied as winding wires. Electrical insulation of polyester is lost over $428 \mathrm{~K}$, which is heatproof temperature of polyester, thus intervals of winding wires have to be coarse to prevent a short between wires. As a result, magnetic field intensity for measurements tends to be low due to small winding number for magnetic field excitation.

In Takeuchi et al.'s method, which is adopted in the present study, a wire consisting of a silver wire (as the inner conductor) and a ceramic braided tube (as the surface insulator) is wound around a test piece for magnetic flux detection and magnetic field excitation. A ceramic braided tube keeps its electrical insulation up to $1473 \mathrm{~K}$, thus high magnetic field can be applied in measurements.

In this study, the two ends of the wire were connected to a DC recording fluxmeter (TRF-5A, Toei Industry Co., Ltd.).
The temperature of the test piece was controlled from room temperature to $1048 \mathrm{~K}\left(775^{\circ} \mathrm{C}\right)$ with an electric furnace. Two test pieces (A and $\mathrm{B}$ ) were placed close together in the furnace: test piece A for B-H curve measurements and test piece $\mathrm{B}$ for the measurement of the actual temperature of the test piece. The end of a thermocouple was welded to the surface of test piece B. The B-H curves were measured in the magnetic field intensity range of $\pm 8000 \mathrm{~A} / \mathrm{m}$.

\subsection{Measurement of Phase Transformation Tempera- tures}

Andres et al. measured phase transformation temperatures using the dilatation curves of steels. ${ }^{24)}$ This methodology was applied in the present study. The measurements were carried out with a thermo-mechanical simulator (ThermecMastor Z, Fuji Electronic Industrial Co., Ltd.). A schematic diagram of the measurement method is shown in Fig. 2. The test piece had a circular cross-section, and its ends were held by jigs. The test piece was heated via Joule heating under an argon atmosphere to prevent oxidization of the test piece. Heating rates were set at 10, 100, 200, 400, and 500 $\mathrm{K} / \mathrm{s}$ and controlled using a proportional-integral-derivative algorithm. Changes in the diameter of the test piece (i.e., dilatation curve) were measured by a non-contact-type LED displacement meter. The material used was medium carbon steel (JIS S45C).

Phase transformation temperatures, $\mathrm{Ac}_{1}$ and $\mathrm{Ac}_{3}$, were measured based on the dilatation curve, as shown in Fig. 3. The diameter of the test piece expands linearly with temperature for temperatures below $\mathrm{Ac}_{1}$. Then, when the temperature is between $\mathrm{Ac}_{1}$ and $\mathrm{Ac}_{3}$, the dilatation curve becomes non-linear, which is caused by the thermal expansion and volume reduction due to austenitic phase transformation (body-centered cubic to face-centered cubic). After austenitic phase transformation is complete, the dilatation

Table 1. Chemical composition of JIS S45C.

\begin{tabular}{ccccc}
\hline $\mathrm{C}$ & $\mathrm{Si}$ & $\mathrm{Mn}$ & $\mathrm{P}$ & $\mathrm{S}$ \\
\hline 0.44 & 0.16 & 0.64 & 0.013 & 0.016 \\
\hline
\end{tabular}

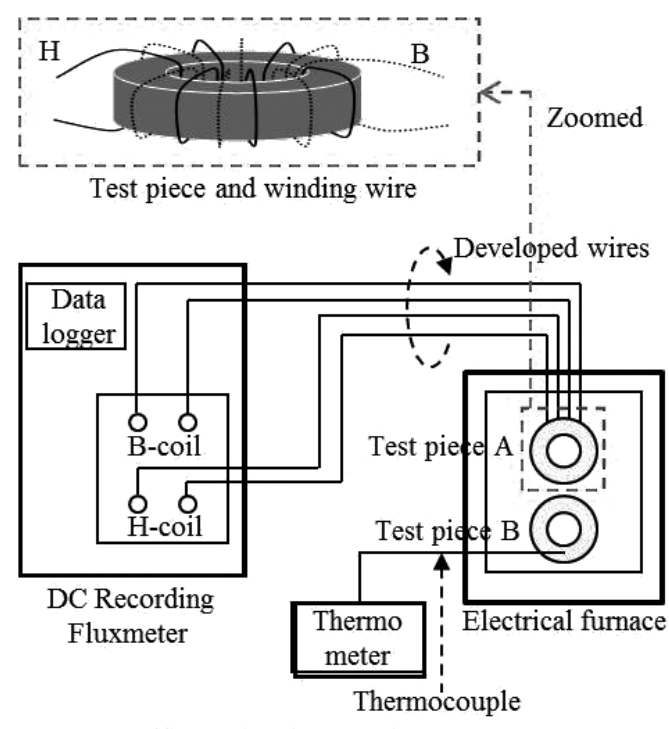

a. Schematic diagram for measurement.

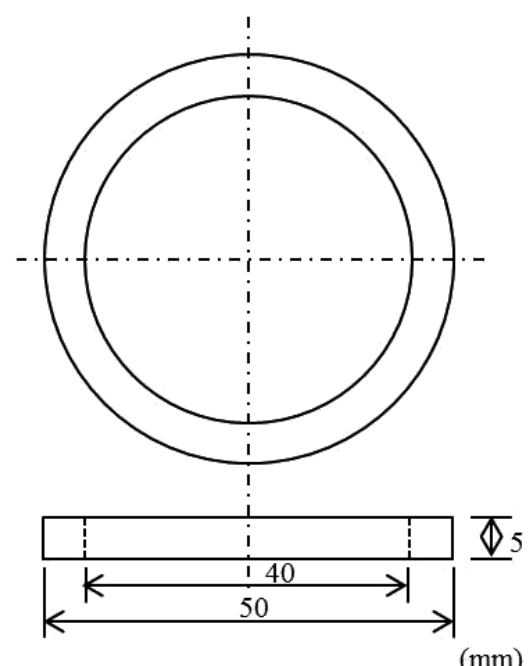

b. Dimensions of a test piece.

Fig. 1. Schematic diagram and dimensions of a test piece for measurements of temperature dependent BH curves. 


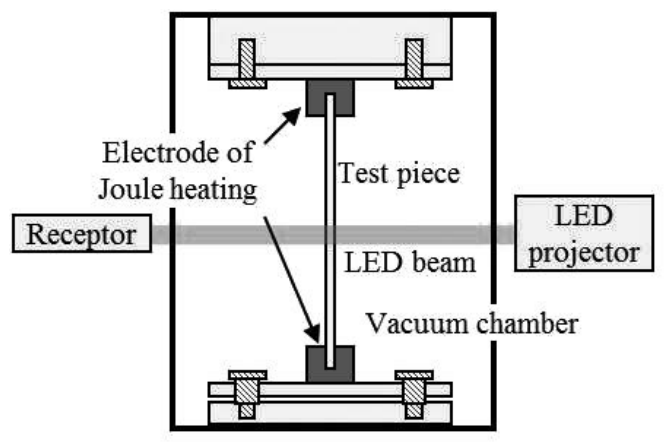

a. Overview (Side view)

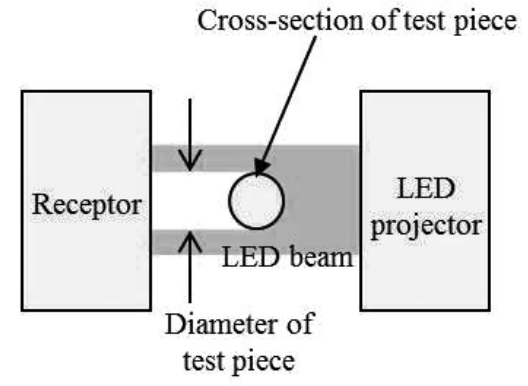

b. Enlarged view of the optical displacement gauge and test piece (Top view)

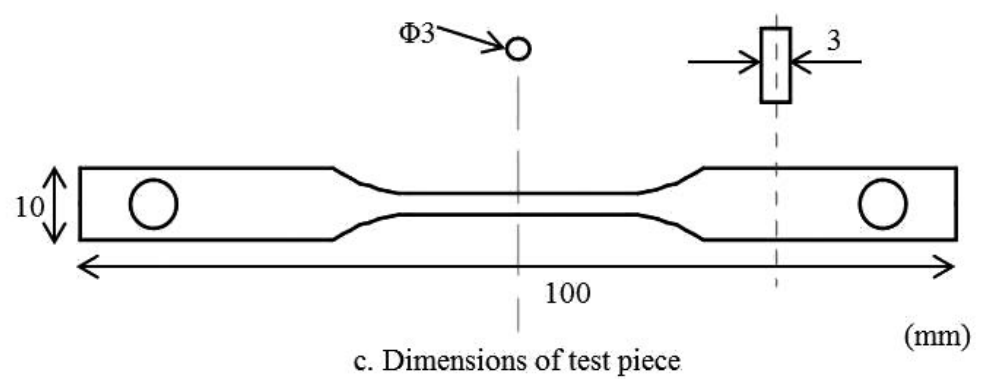

Fig. 2. Schematic diagram of measurement for phase transformation temperature.

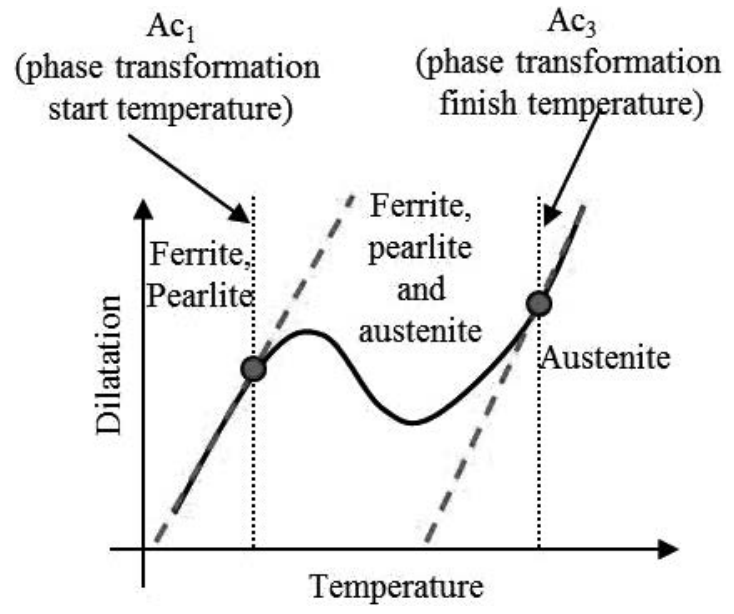

Fig. 3. Identification of phase transformation temperatures, Ac1 and Ac3, from a dilatation curve of a medium carbon steel.

curve becomes linear again. Accordingly, the boundary temperatures between the linear and non-linear sections of the curve were taken as the phase transformation temperatures, $\mathrm{Ac}_{1}$ and $\mathrm{Ac}_{3}$.

\subsection{Induction Heating Experiment}

For comparison with the temperature evolution obtained in the simulation, IH experiments were carried out. The dimensions and positional relationships of the test pieces and heating coils used in the $\mathrm{IH}$ experiments are shown in Fig. 4. Two kinds of test piece were used in the experiments. Test piece A had a columnar shape, and test piece B had a columnar shape with a convex projection. The material used was medium carbon steel (JIS S45C). A one-turn ring-shaped heating coil was applied in the experiments. Type $\mathrm{K}$ thermocouples were welded onto the surface of the test pieces to measure temperature evolution. The coil current was measured using a Rogowski current transducer (CWT-150, Power Electronic Measurements Ltd.). The signals from the thermocouples and the current transducer were recorded using a data logger with a high-speed analog measurement unit (NR-600 and NR-HA08, KEYENCE Co., Ltd.). The data acquisition time was set at $10 \mathrm{~ms}$.

\section{Experimental Results}

The measured temperature-dependent B-H curves are shown in Fig. 5. From room temperature to $973 \mathrm{~K}$, the magnetic flux density decreased gradually with increasing temperature. Above $973 \mathrm{~K}$, the magnetic flux density and permeability decreased drastically. Weak ferromagnetism was observed at $1023 \mathrm{~K}$, but the test piece was paramagnetized at $1048 \mathrm{~K}$, which is higher than the Curie temperature of steel $(1043 \mathrm{~K})$. These results show almost the same temperature-dependent behaviors of pure iron as those reported by Takeuchi et al. ${ }^{23)}$ and described by Maten and Melissen. ${ }^{21)}$ The obtained temperature-dependent B-H curves were thus accurate, making them suitable for simulation.

Figure 6 shows the relationship between the heating rate and changes in phase transformation temperatures measured in Section 2.2. The phase transformation start temperature, $\mathrm{Ac}_{1}$, was almost constant for all heating rates, whereas the phase transformation finish temperature, $\mathrm{Ac}_{3}$, increased with increasing heating rate. These results show the same tendency as that reported for Fe- $0.3 \% \mathrm{C}-0.5 \% \mathrm{Mn}$ steels by Macedo et al. ${ }^{25)}$ The observed behaviors can be explained as follows. When steel reaches the phase transformation start temperature, austenitization starts because the diffusion of carbon atoms is not necessary at the beginning of phase transformation. Therefore, the $\mathrm{Ac}_{1}$ temperature does not depend on the heating rate. As the austenite phase transformation proceeds, the diffusion of carbon atoms becomes predominant. When the steel reaches the phase transformation finish temperature, austenitization does not finish unless the carbon atoms diffuse enough. Accordingly, the $\mathrm{Ac}_{3}$ temperature increases with increasing heating rate. 

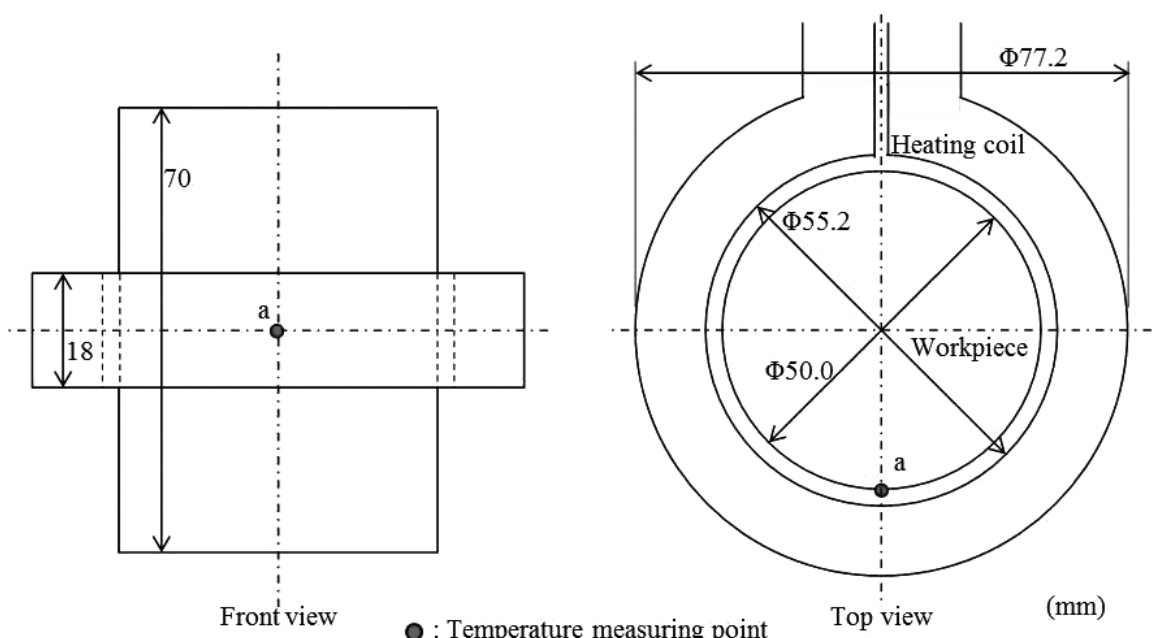

a. Columnar test piece (test piece A)
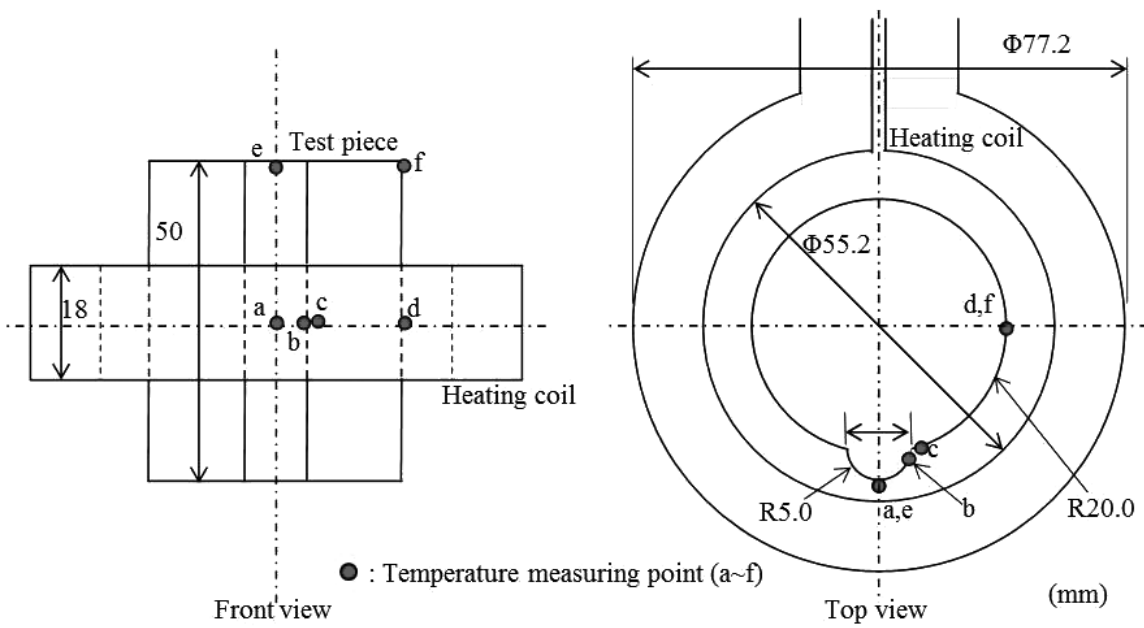

b. Columnar test piece with a convex projection (test piece B)

Fig. 4. Dimensions and positional relationships of test pieces and heating coil used in induction heating experiment.

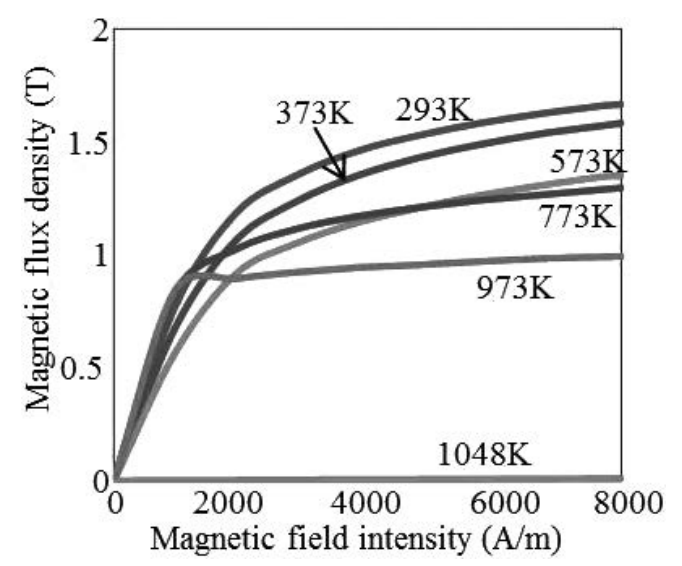

Fig. 5. Temperature dependent B-H curves of JIS S45C.

\section{Simulation Method}

\subsection{Outline of Induction Heating Simulation}

The heating mechanism of IH can be explained in terms of electromagnetic and thermal phenomena as follows. (1) The coil current creates a magnetic flux. (2) The magnetic flux passes through a metallic object. (3) An eddy current is induced around the surface of the metallic object according to Lenz's law. (4) The surface of the metallic object is heated via Joule heating. (5) Heat is conducted from the

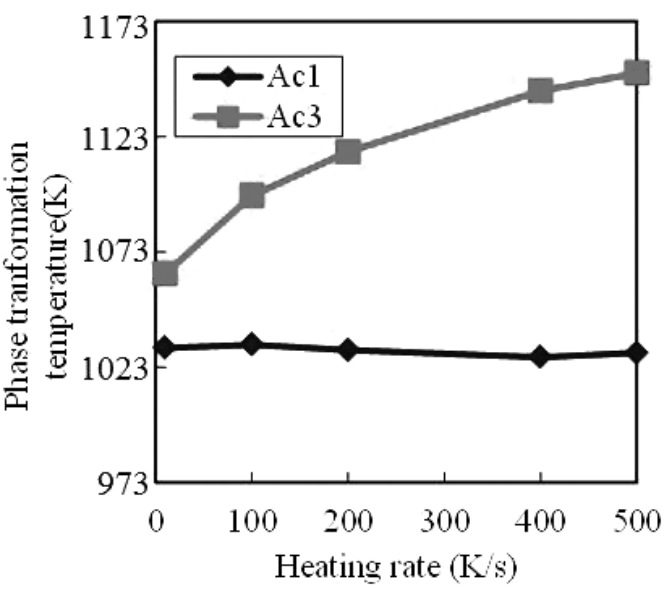

Fig. 6. Relationship between heating rate and phase transformation temperatures of JIS S45C.

surface to the interior. Therefore, IH analysis should be conducted based on coupled electromagnetic and thermal calculations. A schematic diagram of the calculation flow is shown in Fig. 7. The Joule loss distribution of the test piece was first determined using an electromagnetic calculation. In the thermal calculation, the temperature distribution of the test piece was calculated based on the Joule loss distribution. Then, the material properties of the test piece were 


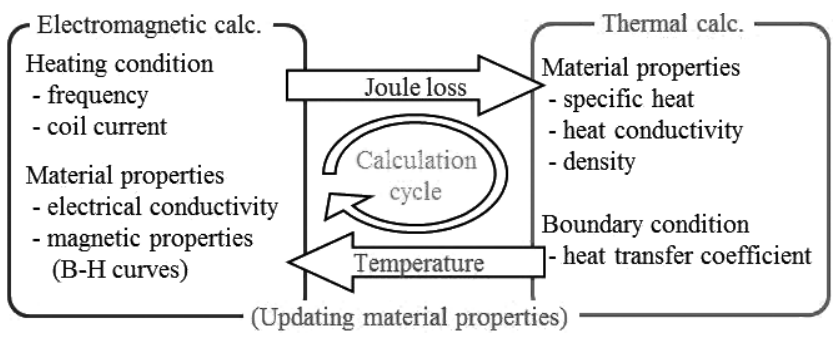

Fig. 7. Schematic diagram of calculation flow for induction heating analysis.

Table 2. Calculation conditions for induction heating analysis.

\begin{tabular}{ccc}
\hline & $\begin{array}{c}\text { Magnetization charasteristics } \\
\text { of workpiece }\end{array}$ & $\begin{array}{c}\text { Phase transformation } \\
\text { of workpiece }\end{array}$ \\
\hline Case1 & BH curve at room temperature & Not considered \\
Case2 & $\begin{array}{c}\text { Temperature dependent BH curves } \\
\text { (From room temperature to 1 048 K) }\end{array}$ & Not considered \\
Case3 & $\begin{array}{c}\text { Temperature dependent BH curves } \\
\text { (From room temperature to 1 048 K) }\end{array}$ & Considered \\
\hline
\end{tabular}

updated based on the temperature distribution, and the Joule loss distribution was calculated again using the updated material properties. Temperature evolution during IH was thus calculated iteratively. The electromagnetic and thermal calculations were done using the commercial finite element method (FEM) software package JMAG (JSOL Co., Ltd.)

Three kinds of IH simulation were conducted with test piece $\mathrm{A}$ to evaluate the effects of considering the temperature-dependent B-H curves and changes in phase transformation temperature under rapid heating on simulation accuracy. The calculation parameters used for the $\mathrm{IH}$ simulation are shown in Table 2. Case 1 uses a conventional analysis. Case 2 considers the temperature-dependent B-H curves. Case 3 considers both the temperature-dependent $\mathrm{B}-\mathrm{H}$ curves and changes in phase transformation temperature under rapid heating. For an additional comparison, case 3 was also conducted using test piece B. Details of the calculation parameters are given in the following sections.

\subsection{Electromagnetic Calculation}

Electromagnetic calculations were conducted using harmonic response simulations. The calculation models are shown in Fig. 8. A two-dimensional axisymmetric FEM model was used for test piece A due to the piece's rotationally symmetric shape. One half of a three-dimensional FEM model was used for test piece B. Air regions were modeled to consider the magnetic flux distribution around the test pieces. Skin meshes were generated around the surface of the test pieces because eddy currents and Joule loss concentrate there due to the skin effect. An example of skin meshes is shown in Fig. 9. The depth of the skin meshes was 0.2 $\mathrm{mm}$ and the number of layers was 5 . The mesh size in the normal mesh region was $1 \mathrm{~mm}$.

In JMAG, a circuit model was coupled with electromagnetic calculations to consider the current density distribution in the heating coil, as it is known that the distribution strongly influences the temperature evolution of heated steel. A circuit model consisting of three elements is shown in Fig. 10. The measured amplitude and frequency of the coil current, shown in Fig. 11, were defined for the current

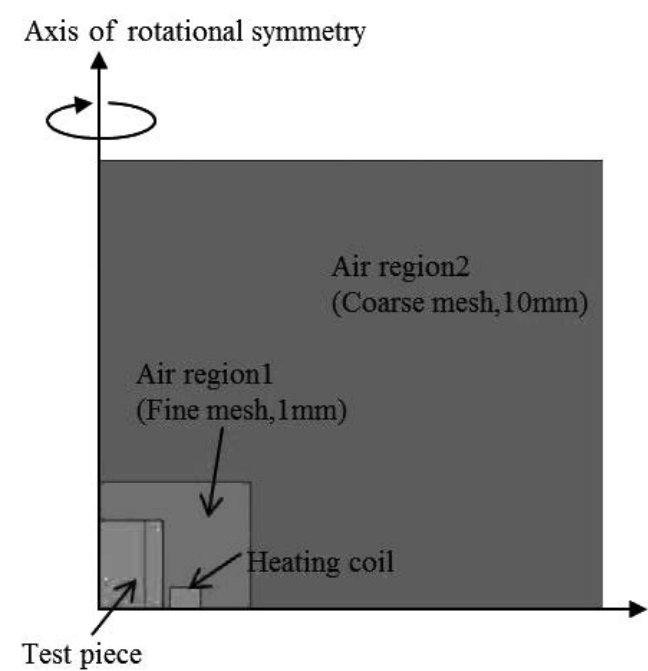

a. Two-dimensional axisymmetric model for test piece A.

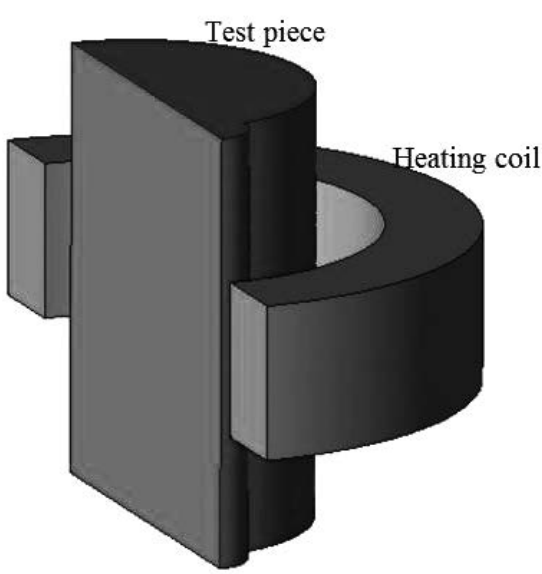

b. Half of three-dimensional model for test piece B.

Fig. 8. Electromagnetic calculation models.

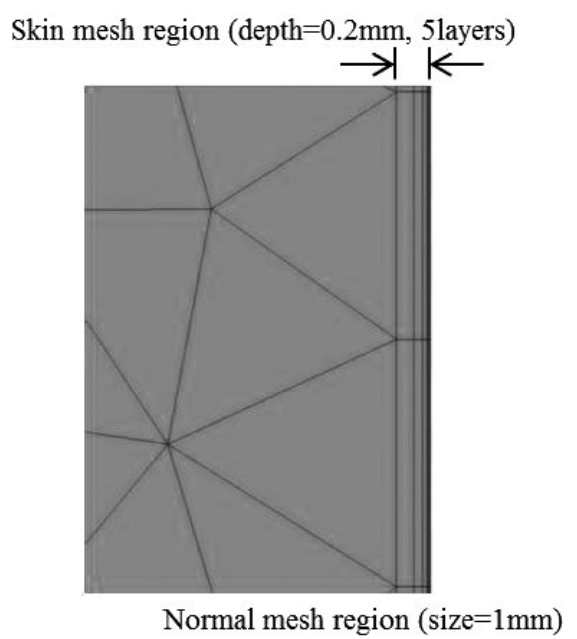

Fig. 9. An example of skin meshes for considering skin effect.

supply element. A FEM conductor element was applied to consider the current density distribution in the heating coil; this element was coupled with the heating coil in the FEM models shown in Fig. 8. The ground element defines zero potential, which was used in circuit calculations.

The measured temperature-dependent B-H curves, shown in Fig. 5, were used in the calculations. The temperaturedependent electrical conductivity (Fig. 12) was also used in the calculations. 


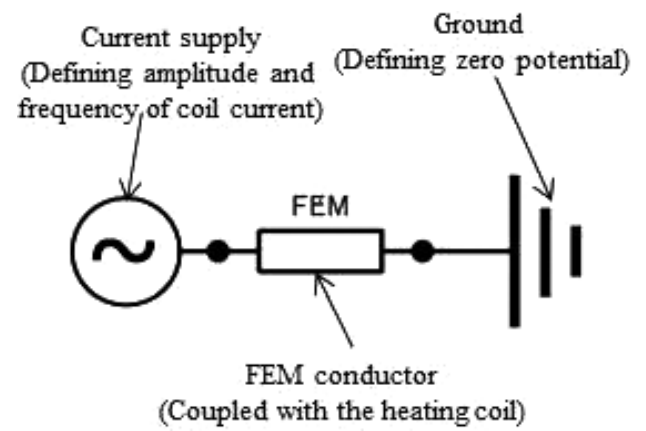

Fig. 10. A circuit model coupled with electromagnetic calculation.

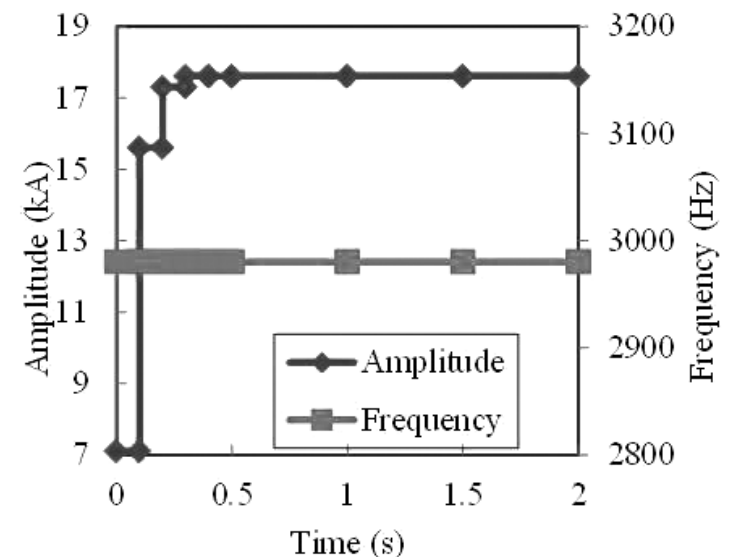

a. Test piece $\mathrm{A}$.

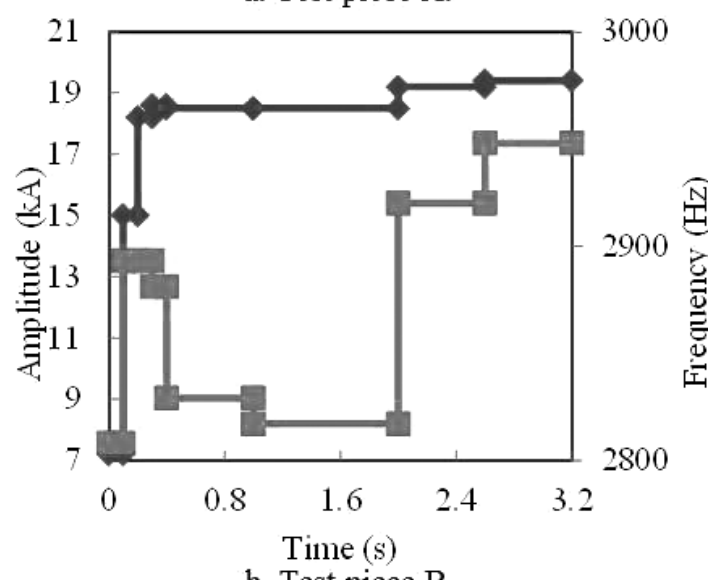

b. Test piece $B$.

Fig. 11. Transition of coil curent and frequency.

\subsection{Thermal Calculation}

The heating coil and air regions were omitted from the thermal calculation models to reduce calculation time. The time step in the thermal calculations was set at $0.1 \mathrm{~s}$.

Changes in phase transformation temperature under rapid heating, measured in Section 2.2, were implemented to the thermal calculation by modification of specific heat data which was input in the thermal calculations. Generally, specific heat is measured by the laser flash method, differential scanning calorimetry, adiabatic method, and so on. The measured specific heat is in equilibrium state due to its slow heating rate. Additionally, latent heat also appears between equilibrium phase transformation temperatures or is not included in measured data. Accordingly, the specific heat used in IH simulation has to be modified to include the transition of the phase transformation temperature and the endothermic reaction due to latent heat under rapid heating.

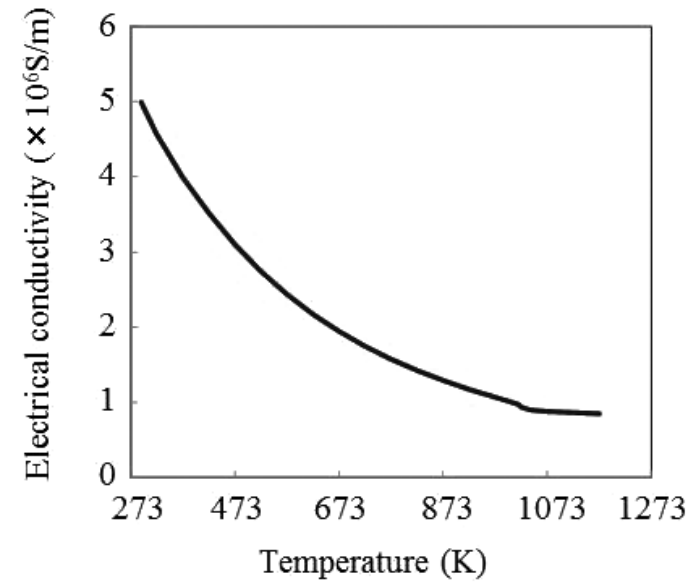

Fig. 12. Temperature dependent electrical conductivity of JIS S45C.

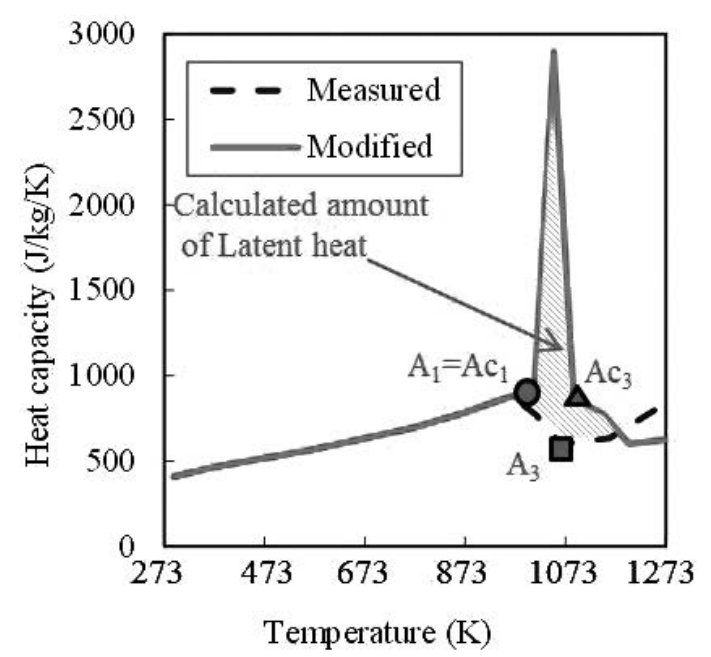

Fig. 13. Modification of specific heat data to consider transition of phase transformation temperature and latent heat (400 $\mathrm{K} / \mathrm{sec})$.

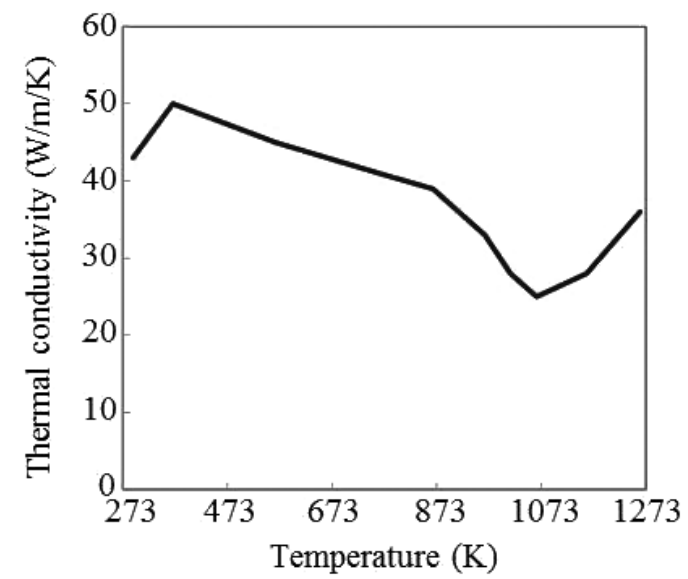

Fig. 14. Temperature dependent thermal conductivity of JIS S45C.

Figure 13 shows the original and modified specific heat data. The broken line is the temperature-dependent specific heat data for JIS S45C measured using the laser flash method and the continuous line is the modified data. The start and finish temperatures of the peak were defined to correspond to the measured $\mathrm{Ac}_{1}$ and $\mathrm{Ac}_{3}$ temperatures, respectively. The height of the peak was defined to make the area sur- 
rounded by the broken and continuous lines correspond to the amount of latent heat. The amount of latent heat, $75 \mathrm{~kJ} /$ $\mathrm{kg}$, was defined according to the measured data reported by Tajima. ${ }^{26)}$ After the peak, the specific heat data for temperatures above the $A_{1}$ temperature were applied to reflect the delay of the phase transformation due to rapid heating.

The temperature-dependent thermal conductivity (Fig. 14) was also considered in the calculations. Density was defined as a constant value of $7800 \mathrm{~kg} / \mathrm{m}^{3}$. A boundary condition was defined on the surface of the test piece to consider heat transfer to air during heating with a heat transfer coefficient of $14.1 \mathrm{~W} / \mathrm{m}^{2} \mathrm{~K}$.

\section{Simulation Results and Discussion}

A comparison between the experimental and calculation results for test piece A is shown in Fig. 15. In case 1, the calculated temperatures are higher than the experimentally measured temperatures for the entire heating process. The calorific values of the test piece must decrease with increasing temperature because the magnetic flux density decreases, as shown in Fig. 5. However, this phenomenon is ignored when the conventional method is used, explaining the higher calculated temperatures.

In case 2, with the application of the temperature-dependent B-H curves, the calculated temperatures shows good agreement with the experimentally measured temperatures for temperatures up to $973 \mathrm{~K}$. The calculated and experimental values diverge at temperatures above $973 \mathrm{~K}$. This error can be decreased by considering the changes in phase transformation temperature under rapid heating, as done in case 3 . In case 3, the maximum error between the experimental and calculated values is approximately $25 \mathrm{~K}$, which is 9 times smaller than that for a conventional calculation (case 1).

A comparison between the experimental and calculated temperatures for test piece B is shown in Fig. 16. Temperature evolution at measurement points $a-d$ was almost the same up to $973 \mathrm{~K}$. The experimental and calculated temperatures at these points diverged above $973 \mathrm{~K}$. The rate of temperature evolution at point $a$ slightly decreased for a few subseconds. The temperatures at the other points increased at different rates. The heating rate at point $b$ was relatively slow, and that at point $c$ was the fastest. The difference between the temperatures at points $a$ and $c$ was approximately $270 \mathrm{~K}$ at the end of heating. The temperatures at points $e$ and $f$ increased monotonically, and thus it is assumed

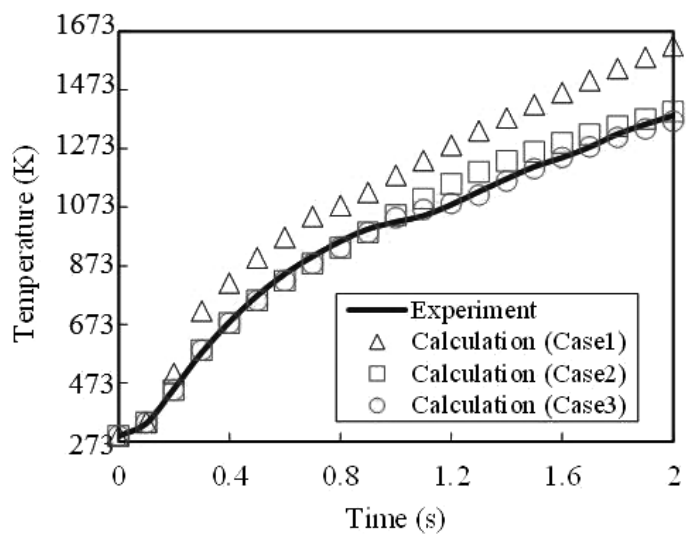

a. Transition of surface temperature.

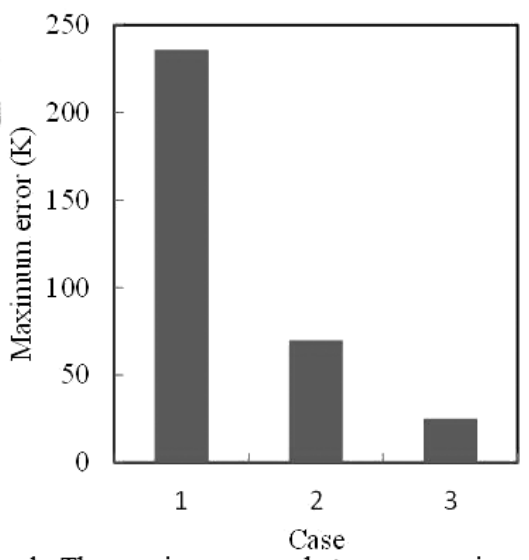

b. The maximum error between experimental and calculation result.

Fig. 15. Comparison between experimental and calculation result for test piece A.

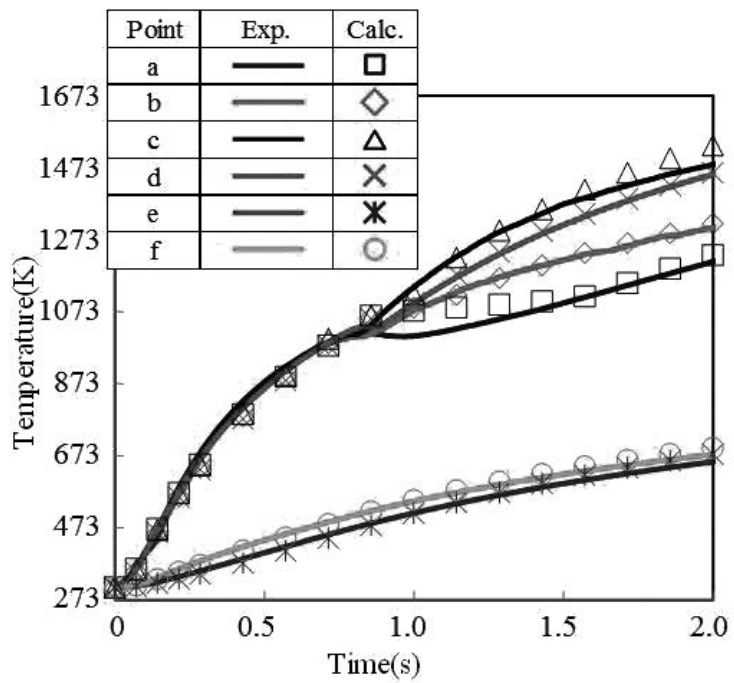

a. Transition of surface temperatures.

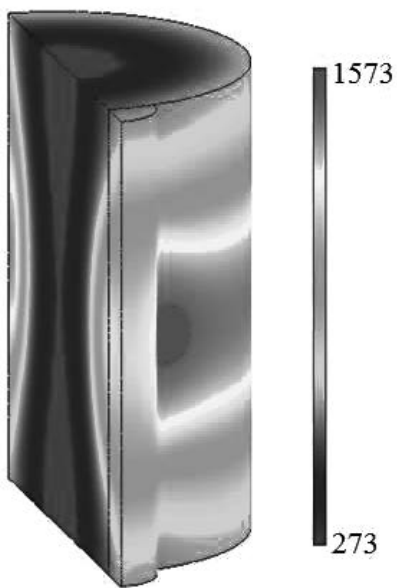

$(\mathrm{K})$

Fig. 16. Comparison between experimental and calculation results for test piece B. 

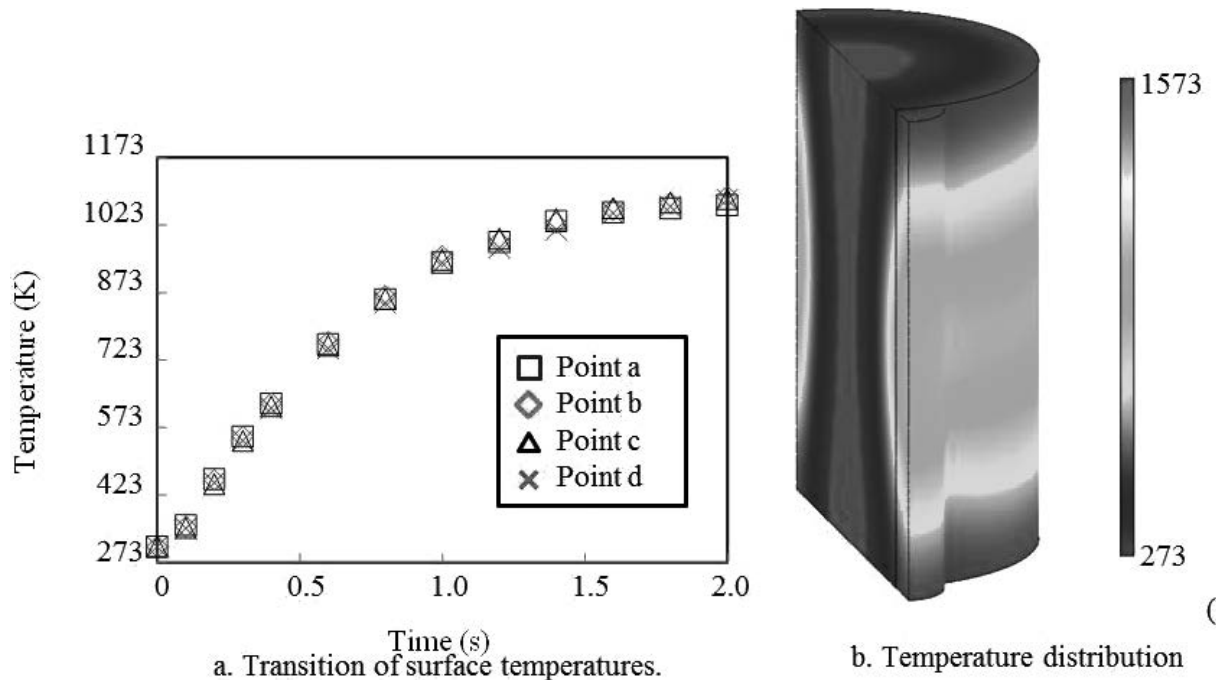

b. Temperature distribution

Fig. 18. Calculation results for the purpose of temperature equalization of test piece B.

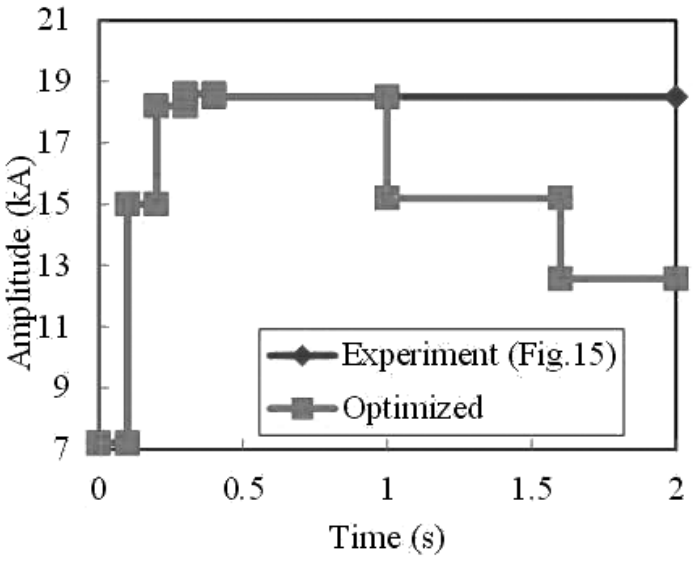

Fig. 17. Time transition of coil current amplitude.

that these points were heated due to heat conduction from a high-temperature region. The accuracy for point $a$ drops at around $1073 \mathrm{~K}$. Nevertheless, the calculations captured the complicated temperature evolution behavior at all points.

Additionally, a non-uniform temperature distribution, such as that shown in Fig. 16(b), is not desirable in actual heat treatment. Thus, the developed simulation was used to determine the optimal coil current amplitude for achieving a uniform temperature distribution around the convex projection of test piece $B$. The optimized time transition of the coil current amplitude and the calculation results are shown in Figs. 17 and 18. The temperature dispersion around the convex projection became quite small with twostep decreasing of the coil current amplitude after $1.0 \mathrm{~s}$ of heating. The maximum difference between the temperatures at points $a-d$ was $20.3 \mathrm{~K}$.

\section{Conclusion}

IH analysis with consideration of the temperature-dependent B-H curves and changes in phase transformation temperature under rapid heating was conducted. The accuracy of the calculation results from room temperature to around $973 \mathrm{~K}$ was significantly improved by considering the temperature-dependent B-H curves. At temperatures of above $973 \mathrm{~K}$, consideration of changes in phase transformation temperatures and latent heat under rapid heating effectively improved the accuracy. The complicated temperature evolution and distributions of a test piece with a convex projection could be simulated with good agreement. The optimal heating parameters for achieving a uniform temperature distribution were determined.

This simulation could be applied to the design of heating coils and the setting of heating parameters, such as coil current amplitude, frequency, and heating time, without trial and error.

\section{REFERENCES}

1) V. Rudnev, D. Loveless and R. L. Cook: Handbook of Induction Heating, CRC Press, Baca Raton, (2017), 9.

2) Y. Misaka, K. Kawasaki, J. Komotori and M. Shimizu: Mater. Trans., 45 (2004), 2930.

3) S. Fujikawa: J. Jpn. Soc. Technol. Plast., 52 (2011), 148 (in Japanese).

4) H. K. Jung and C. G. Kang: J. Mater. Process. Technol., 120 (2002), 355.

5) B. Xiong, C. Cai, H. Wan and B. Lu: Mater. Des., 32 (2011), 2978.

6) K. Nakamoto, M. Anzai and S. Matsumoto: J. Jpn. Soc. Precis. Eng., 74 (2008), 543 (in Japanese)

7) C. Carpenter: J. Petrol. Technol., 69 (2017), 75

8) M. H. Tavakoli: Cryst. Growth Des., 8 (2008), 483.

9) K. Zhou and T. Zhang: Mater. Trans., 58 (2017), 663.

10) M. Chen, L. Yuan and S. Liu: Sens. Actuators A-Phys., 133 (2007), 266.

11) H. Tomita: J. Adhes. Soc. Jpn., 39 (2003), 24 (in Japanese).

12) G. Zhang, H. Cui, H. Zhang and G. Cheng: Tribol. Int., 120 (2018), 226.

13) S. Kikuchi, S. Iwamae, H. Akebono, J. Komotori and K. Kadota: Surf. Coat. Technol., 334 (2018), 189.

14) E. Pongracz, J. Y. Mella, R. Keiski, P. Phillips, P. Tanskanen and J. Kaakinen: J. Solid Waste Technol. Manag., 31 (2005), 102.

15) Y. Zouambia, K. Y. Ettoumi, M. Krea and N. M. Mostefa: Arab. J. Chem., 10 (2017), 480.

16) K. Kawasaki: J. Jpn. Soc. Heat Treat., 37 (1997), 133 (in Japanese).

17) K. Miyachika, S. Oda, M. Kato, H. Tsuboi and H. Fujio: Trans. Jpn. Soc. Mech. Eng. C, 64 (1998), 375 (in Japanese).

18) M. Takagaki and Y. Toi: Trans. Jpn. Soc. Mech. Eng. A, 71 (2005), 47 (in Japanese).

19) I. R. Ciric, F. I. Hantila and M. Maricaru: IEEE Trans. Magn., 44 (2008), 1190.

20) F. Ikuta and T. Horino: J. Jpn. Soc. Heat Treat., 42 (2002), 359 (in Japanese).

21) E. J. W. ter Maten and J. B. M. Melissen: IEEE Trans. Magn., 28 (1992), 1287.

22) T. Miwa and N. Iguchi: J. Jpn. Inst. Met., 37 (1973), 945 (in Japanese).

23) H. Takeuchi, Y. Yogo, T. Hattori, T. Tajima and T. Ishikawa: ISIJ Int., 57 (2017), 1883.

24) C. G. de Andrés, F. G. Caballero, C. Capdevila and L. F. Álvarez: Mater. Charact., 48 (2002), 101.

25) M. Q. Macedo, A. B. Cota and F. G. S. Araujo: Rem: Rev. Esc. Minas, 64 (2011), 163.

26) M. Tajima: Tetsu-to-Hagané, 84 (1998), 547 (in Japanese). 